\section{P-252 BREAKING DOWN THE BARRIERS TO MANDATORY TRAINING}

Jacquie Pamphilon, Jennifer Chandler. St Helena Hospice, Colchester, UK

\subsection{6/bmjspcare-2018-hospiceabs.277}

Background Employees and volunteers did not understand the importance and necessity of mandatory training, resulting in a lack of knowledge to support a safe and secure working environment. 'Good compliance training should help people understand why it's so important.' (Scott, Mannion, Davies et al., 2003).

As an organisation we care about the wellbeing of our staff and volunteers, we recognised that the risk of non-compliance left our employees and volunteers exposed to possible harm and danger. The Learning and Development Team analysed why the completion of mandatory training was failing. The results directed us to change working practices and create a culture where mandatory training is recognised as a benefit and not a negative.

Aims

- Understand why compliance was low

- Learning from previous failures

- Discussion of incidents

- Make mandatory training relevant to role

- Inform the benefits of mandatory training

- Overcome barriers

- Create and maintain a culture change

- Uphold compliance figures.

Methods

- Created policy

- Introduced mandatory training on induction

- Line manager support during induction period

- Line managers supporting annual updates

- Scheduling within rotas for clinical staff

- Learning and Development one-to-one support.

Results June 2015 - demonstrated 30\% average compliance rate. June 2018 - demonstrated 92\% average compliance rate (this will increase with planned scheduling).

Conclusion As an organisation our goal is to achieve and maintain an average of $95 \%$ compliance rate for all mandatory training. The senior management understand the rationale and have endorsed the culture change. This helped with motivating teams, and individuals realising the benefits of mandatory training. With processes that have been implemented, the new scheduling, and continuing to embed the culture of embracing mandatory training, we expect high compliance to become common practice.

\section{P-253 DEALING WITH DYING EDUCATIONAL WORKSHOPS}

Emma Mazerolle, Ross Chirgwin. St Helena Hospice, Colchester, UK

\subsection{6/bmjspcare-2018-hospiceabs. 278}

Background Dealing with Dying is a difficult but important aspect of palliative and end of life care. In response to feedback from patients' families, who felt they were not prepared with the realities of people dying and what was expected, a number of educational workshops were developed to address this learning need.
Aims The workshops were focused on ensuring families were aware of what realistic choices were available without 'unrealistic' or 'romanticised' expectations. These were initially developed for families, however, the workshops have also been adapted and delivered to hospice staff, volunteers and other healthcare professionals who wanted further insight into the dying process. The information we provided was factual and quite hard hitting to help prepare families who are caring for loved ones at the end of life.

Method In total ten workshops were delivered for both families and healthcare professionals and were based on three key themes:

- Realistic choices. This included what services were available, the importance of advance care planning, challenges in care provision and adapting the home to meet the patient's needs

- Process of dying. This provided information on potential symptoms patients may develop and how these could be managed within the community

- When someone dies at home. This included what to do after someone dies, practical measures including what the process is following death and into the future.

Results In excess of 100 families and healthcare professionals have engaged with these workshops, which have been positively evaluated. As part of this process these workshops have been adapted in response to constructive feedback.

Conclusion These workshops helped facilitate discussion and promote families and healthcare professionals not only to talk more openly about death and dying, but also to increase knowledge and confidence around this sensitive subject.

\section{P-254 ONLINE UPSKILLING IN MOTOR NEURONE DISEASE FOR CARE HOMES... AND HOSPICES?}

Jennifer Bedford. MND Association, Northampton, UK

\subsection{6/bmjspcare-2018-hospiceabs.279}

Background MND Association regional staff were often asked to deliver MND talks in care homes. Frequently on arriving to present, the number of care workers was much fewer than expected or, no-one was available at all due to work pressures.

Aims To develop a resource:

- accessible at point of need

- to meet the learning requirements of care workers or those in similar roles

- flexible around work commitments.

To review its effectiveness.

Methods

- Project development group with representatives from MDT professions and hospice sector

- Collaborative face to face and remote content development

- Content to be practical advice, based on professional opinion

- Flexible structure to address issues of time poverty and staff turnover

- Free

- Each section to cover a symptom management/knowledge area

- Opportunity to complete over a number of days/weeks

- Certificate of completion

- Reviewed via an online survey and course monitoring data. 
Results Development of a free online course hosted by University of Northampton. Participants:

- $97 \%$ agreed/strongly agreed that the module had been organised in a way that helped them to learn

- $68 \%$ felt that they had significantly increased their knowledge of MND and a further 30\% believed they had increased their knowledge

- Following the course 94\% felt more confident about caring for someone living with MND.

Reasons for completing the course varied: $47 \%$ indicated they were caring for someone with MND, and 32\% were undertaking it purely for professional development. A significant proportion of completions were outside core hours: $31 \%$ 7 pm - midnight and 11\% midnight - 7 am; a requirement not easily accommodated by training. The course has been used to provide wholesale staff training.

Conclusion This module has approximately 700 completions to date. It provides a comprehensive, practical introduction to MND and has been well received by participants. It could usefully be employed within hospices to support the delivery of outstanding care.

\section{P-255 THE IMPLEMENTATION OF PROJECT ECHO INTO NORTH EAST ESSEX}

Caroline Vince, Imelda Hodgkinson, Christine Aylott, Karen Chumbley, Jacquie Pamphilon, Jennifer Chandler. St Helena Hospice, Colchester, UK

10.1136/bmjspcare-2018-hospiceabs.280

Background Project ECHO ${ }^{\circledR}$ (Extension of Community Healthcare Outcomes) is a virtual environment learning tool, used within a community of practice among care providers, to facilitate a more comprehensive seamless experience for care staff, patients and families.

Aim The concept of ECHO in this instance is being utilised to disseminate knowledge and best practice within the palliative care community to enable cohesive working. This united approach lends itself to problem solving and improving the quality of decision making within the healthcare sector with emphasis on collaboration and shared focus, to enhance standards of care, in a dynamic and revolutionary manner.

Method Utilising the Gold Standards Framework workshops for nursing and residential homes, we introduced the concept of ECHO by demonstrating a video example plus an in-house demonstration. As a consequence, we gained buy-in and will run a pilot dividing the nursing and residential homes into two groups of eight and delivering six ECHO sessions over a period of six months from July 2018.

Results These sessions will be based on the identified learning needs of the 16 nursing and residential homes. The first three sessions will be based on communication, symptom management at the end of life and advance care planning. A guest speaker will provide a 20 min presentation on the chosen subject which will set the tone for the remainder of the discussion. Two of the nursing and residential homes will provide case studies relating to the theme of the session with active participation from the expert panel at the hospice hub and all participants.

Conclusion From initial observation, the idea of ECHO has captured the enthusiasm and willingness of shared learning to improve patient experience with 16 out of the 24 nursing and residential homes voicing an interest in engaging with the opportunity to participate in ECHO.

\section{P-256 DESIGNING AN E-ELCA LEARNING PATH FOR SPECIALISTS IN PALLIATIVE CARE}

${ }^{1,2,3}$ Richard Kitchen, ${ }^{4}$ Emily Curran. 'University Hospitals of Coventry and Warwickshire, Coventry, UK; ${ }^{2}$ The Myton Hospices, Warwick, UK; ${ }^{3}$ The Association for Palliative Medicine, Fareham, UK; ${ }^{4}$ Health Education Yorkshire and the Humber, Leeds, UK

\subsection{6/bmjspcare-2018-hospiceabs.281}

Background e-ELCA (end of life care for all) is an e-learning programme from e-Learning for Health, delivering palliative and end of life care education. It was originally developed to support the Department of Health's End of Life Care Strategy, being designed to deliver education to the wider NHS workforce. More recently, there has also been a focus on offering educational opportunities to specialists in palliative care. Of note, e-ELCA is comprised of over 160 sessions and utilises 'learning paths' to allow users to identify sessions that will be helpful for their learning.

Aim To design an e-ELCA learning path for specialists in palliative care. This would allow this group to easily identify e-ELCA sessions that are relevant for their own learning.

Methods The JRCPTB (Joint Royal College of Physicians Training Board, 2014) speciality training curriculum for palliative medicine is used in the training of palliative medicine specialty registrars. The curriculum contains many sections including physical care, communication and ethics. Educational resources that support this curriculum will be helpful for registrars, but are also likely to be relevant for other specialists in palliative care. Therefore, e-ELCA sessions were mapped to the JRCPTB speciality training curriculum for palliative medicine (2010 with amendments 2014)1. This process was carried out by a specialty registrar in palliative medicine, with this work then reviewed by the e-ELCA clinical lead.

Results Initially 34 e-ELCA sessions were identified that mapped to the curriculum. Following further review, 10 of these were deemed to too basic in content for specialists in palliative care. The 24 sessions that remained comprised the final version of the learning path. This is now available on the e-ELCA website.

Conclusions The JRCPTB speciality training curriculum for palliative medicine was used to identify e-ELCA sessions that specialists in palliative care could use for their own education.

\section{P-257 A REVIEW OF THE (QELCA)@ (QUALITY AT END OF LIFE CARE FOR ALL) PROGRAMME ADAPTED FOR LEADERS AT ST CHRISTOPHER'S HOSPICE}

Anne Nash, Kim Briggs, Julie O'Neill. St Christopher's Hospice, London, UK

10.1136/bmjspcare-2018-hospiceabs.282

This programme, funded by the Burdett Trust, used an adapted version of the (QELCA) C) course. Its aim was to develop the leadership skills of inpatient managers. The group visited and observed external NHS leaders in both hospital 